\title{
Transit timing variations, radial velocities and long-term dynamical stability of the system Kepler-410*
}

\author{
Pavol Gajdoš $\check{1}^{\dagger}$, Martin Vaňko², Theodor Pribulla ${ }^{2}$, Daniel Dupkala ${ }^{3,4}$, Ján Šubjak ${ }^{3,4}$, \\ Marek Skarka ${ }^{3,5}$, Petr Kabáth ${ }^{3}$, Lubomír Hambálek ${ }^{2}$, Štefan Parimucha ${ }^{1}$ \\ ${ }^{1}$ Institute of Physics, Faculty of Science, Pavol Jozef Šafárik University, Košice, Slovakia \\ ${ }^{2}$ Astronomical Institute, Slovak Academy of Sciences, 05960 Tatranská Lomnica, Slovakia \\ ${ }^{3}$ Astronomical Institute, Czech Academy of Sciences, Fričova 298, 25165, Ondřejov, Czech Republic \\ ${ }^{4}$ Astronomical Institute of Charles University, V Holešovičkách 2, 180 00, Praha, Czech Republic \\ ${ }^{5}$ Department of Theoretical Physics and Astrophysics, Masaryk Univerzity, Kotlářská 2, 61137 Brno, Czech Republic
}

Accepted 2019 January 22. Received 2019 January 21; in original form 2018 December 14.

\begin{abstract}
Transit timing variations of Kepler-410Ab were already reported in a few papers. Their semi-amplitude is about 14.5 minutes. In our previous paper, we found that the transit timing variations could be caused by the presence of a stellar companion in this system. Our main motivation for this paper was to investigate variation in a radial-velocity curve generated by this additional star in the system. We performed spectroscopic observation of Kepler-410 using three telescopes in Slovakia and Czech Republic. Using the cross-correlation function, we measured the radial velocities of the star Kepler-410A. We did not observe any periodic variation in a radial-velocity curve. Therefore, we rejected our previous hypothesis about additional stellar companion in the Kepler-410 system. We ran different numerical simulations to study meanmotion resonances with Kepler-410Ab. Observed transit timing variations could be also explained by the presence of a small planet near to mean-motion resonance 2:3 with Kepler-410Ab. This resonance is stable on a long-time scale. We also looked for stable regions in the Kepler-410 system where another planet could exist for a long time.
\end{abstract}

Key words: planetary systems - planets and satellites: dynamical evolution and stability - techniques: radial velocities - methods: numerical - stars: individual: Kepler410

\section{INTRODUCTION}

The transiting exoplanet Kepler-410Ab, a Neptune-sized planet on the 17.8336 days orbit, was discovered in 2013 by Kepler telescope and confirmed by Van Eylen et al. (2014). The host star Kepler-410A (KIC 8866102, KOI-42, HD 175289) is a young $2.76 \pm 0.54$ Gyr old star with radius $1.352 \pm 0.010 \mathrm{R}_{\odot}$, mass $1.214 \pm 0.033 \mathrm{M}_{\odot}$, a spectral type F6IV and $V$ magnitude 9.5 (Molenda-Żakowicz et al. 2013). The second GAIA Data Release (Gaia Collaboration et al. 2018) gives a distance of $148.16 \pm 0.49 \mathrm{pc}$.

Using adaptive optics, Adams et al. (2012) distinguished a low-mass stellar companion Kepler-410B separated by an angular distance of 1.63 arcsec. Van Eylen et al. (2014) found

\footnotetext{
* This article is based on the data collected with Perek 2-m telescope.

† E-mail: pavol.gajdos@student.upjs.sk
}

that this star is probably a red dwarf and ruled it out as a host star for the exoplanet.

We analysed transit timing variations (TTVs) in this system in our previous paper (Gajdoš et al. 2017). We found periodic variations with semi-amplitude of about 14.5 minutes which we explained using a model of light-time effect (LiTE) (Irwin 1952a) and perturbing model given by Agol et al. (2005), by the presence of another stellar-mass $\left(1-2 \mathrm{M}_{\odot}\right)$ object in Kepler-410 system.

The aim of this paper is to observe variations in a radialvelocity curve caused by this stellar companion and to analyse them. A second motivation of the present study was to perform simulations leading to the generation of stability maps for the system. We also studied the possibility of mean-motion resonances with Kepler-410Ab and a stability of them.

In Section 2 we summarize the results of previous studies about TTVs of Kepler-410Ab. In Section 3 we present our spectroscopic observations and radial velocities of the sys- 


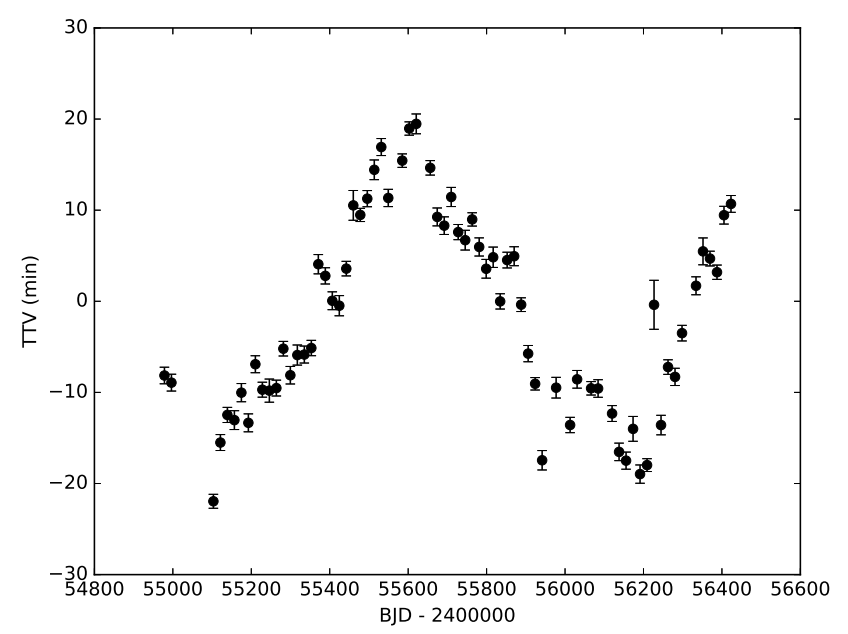

Figure 1. Transit timing variations of Kepler-410Ab presented in our previous paper (Gajdoš et al. 2017).

tem. We analyse resonant perturbation in Section 4 Section 5 is concerned with the long-term stability of the system. Our results are discussed in Section 6 .

\section{TRANSIT TIMING VARIATIONS}

TTVs of Kepler-410Ab were for the first time reported by Mazeh et al. (2013). They studied the TTVs using a sinusoidal model and found a TTV semi-amplitude of $13.91 \pm 0.91$ minutes and a period of about 960 days. In a subsequent analysis, Van Eylen et al. (2014) used a 'zigzag' model. They obtained a slightly larger semi-amplitude of 16.5 minutes, and a period of 957 days. They also noted that the shape of the TTVs in not sinusoidal, which could be caused by an eccentric orbit of Kepler-410Ab. Holczer et al. (2016) found out TTVs with semi-amplitude of $15.59 \pm 0.49$ minutes and a period of $960.6 \pm 9.2$ days.

In our previous study (Gajdoš et al. 2017), we found periodic variations with semi-amplitude of about 14.5 minutes and period of about 972 days (see Figure 11). We used two different models to explain the observed variations. The first one was a model of light-time effect (Irwin 1952a). Using the LiTE solution, we arrived at the mass function $f\left(M_{3}\right)=0.879 \pm 0.030 \mathrm{M}_{\odot}$. For a coplanar orbit, we got the minimal mass of the body $M_{3}=2.151 \pm 0.078 \mathrm{M}_{\odot}$. If we consider a main-sequence star, this would correspond to the spectral type A4. Star with such luminosity was not detected in spectra obtained by Molenda-Żakowicz et al. (2013) nor in our new spectra (see Section 3). Moreover, no signs of eclipses with $\sim 970$-day period were found during the $\sim 1460$-day long observation by Kepler. Thus, we concluded that the perturbing body could be a non-eclipsing binary star. Kepler-410 is a quadruple star with one planet (orbiting Kepler-410A) in this scenario.

We also used an analytical approximation of the perturbation model given by Agol et al. (2005, their eq. 25). This method was originally developed for the analysis of multiple planetary systems but is not constrained to any limit of mass of the perturbing body. We assumed coplanar orbits and calculated the mass of the third body
$M_{3}=0.906 \pm 0.155 \mathrm{M}_{\odot}$. If this model is correct, Kepler-410 is a triple star system with one planet.

\section{SPECTROSCOPIC OBSERVATIONS AND RADIAL VELOCITIES}

TTVs models proposed in our paper predict radial-velocity (RV) changes with semi-amplitude $\sim 25-30 \mathrm{~km} / \mathrm{s}$ (Irwin 1952b). The period of the variation would be the same as the period of possible stellar companion (i.e. $\sim 972$ days). The precision at the level of $1 \mathrm{~km} / \mathrm{s}$ is more than sufficient for detecting this variation.

Our spectroscopic observations of Kepler-410 started in 2016 with a $60-\mathrm{cm}$ telescope at Stará Lesná Observatory. During years 2016 - 2018 we made overall 60 RV measurements (listed in Table 1) using three telescopes.

We obtained 34 spectra at Stará Lesná Observatory. The Stará Lesná Observatory $\left(20^{\circ} 17^{\prime} 21^{\prime \prime} \mathrm{E}, 49^{\circ} 09^{\prime} 06^{\prime \prime} \mathrm{N}\right.$, 810 meters a.s.l.) is part of the Astronomical Institute of the Slovak Academy of Sciences. It is equipped with 60$\mathrm{cm}$ (f/12.5) Zeiss reflecting telescope with fiber-fed échelle spectrograph eShel (Pribulla et al. 2015). The spectra consisting of 24 orders cover the wavelength range from 4150 to $7600 \AA$. The resolving power of the spectrograph is $R=10000-12000$. The ThAr calibration unit provides about $100 \mathrm{~m} / \mathrm{s}$ radial-velocity accuracy. Reduction pipeline using IRAF (Tody 1993) has been described by Pribulla et al. (2015).

Kepler-410 system was also observed on 22 different nights during 2017 till 2018 period in Ondřejov Observatory $\left(14^{\circ} 46^{\prime} 52^{\prime \prime} \mathrm{E}, 49^{\circ} 54^{\prime} 55^{\prime \prime} \mathrm{N}, 525\right.$ meters a.s.l.). We obtained spectra with OES spectrograph installed at 2-m Perek telescope. OES is an échelle spectrograph with resolving power $R=44000$ sensitive between $3600-9500 \AA$. OES is using ThAr spectra for wavelength calibration. The more detailed description is given in Kabath et al. (2018). The spectra were reduced with IRAF.

We obtained also four spectra in 2018 with a $1.3 \mathrm{~m}$, f/8.36, Nasmyth-Cassegrain telescope equipped with a fiberfed échelle spectrograph at Skalnaté Pleso Observatory $\left(20^{\circ} 14^{\prime} 02^{\prime \prime} \mathrm{E}, 49^{\circ} 11^{\prime} 22^{\prime \prime} \mathrm{N}, 1786\right.$ meters a.s.l.). The spectrograph follows the MUSICOS design (Baudrand \& Bohm 1992). The spectra were recorded by an Andor iKon 936 DHZ CCD camera, with a $2048 \times 2048$ array, $15 \mu$ m square pixels, $2.7 \mathrm{e}^{-}$read-out noise and gain close to unity. The spectral range of the instrument is $4250-7375 \AA$ (56 échelle orders) with the maximum resolution of $R=38000$. Reduction pipeline is the same as in the case of spectra from Stará Lesná Observatory.

In all collected spectra, we could detect spectral lines only from one stellar source (Kepler-410A). A dwarf star Kepler-410B is too faint to be detected using our spectra with low SNR obtained by small telescopes. No spectroscopic signal from hypothetical star Kepler-410C (one star or close binary), proposed in our TTV analysis (see Section 2), was observed. It is the first observational evidence that TTVs are caused by a different mechanism.

We measured the radial velocity of Kepler-410 using cross-correlation function in iSpec (Blanco-Cuaresma et al. 2014). We have selected only spectral region around magnesium triplet $(5000-5500 \AA)$. As a template, we used a 
Table 1. Radial velocity measurements from observatories: Ond. - Ondřejov Observatory, SL - Stará Lesná Observatory, SP - Skalnaté Pleso Observatory

\begin{tabular}{|c|c|c|c|c|}
\hline HJD-2400000 & $\mathrm{RV}(\mathrm{km} / \mathrm{s})$ & Error $(\mathrm{km} / \mathrm{s})$ & SNR & Obs. \\
\hline 57561.4897 & -40.40 & 0.37 & 33 & SL \\
\hline 57563.4849 & -40.17 & 0.37 & 28 & SL \\
\hline 57563.5207 & -40.52 & 0.37 & 22 & SL \\
\hline 57575.3651 & -40.68 & 0.35 & 22 & SL \\
\hline 57608.4225 & -41.04 & 0.37 & 29 & SL \\
\hline 57609.3982 & -40.70 & 0.37 & 35 & $\mathrm{SL}$ \\
\hline 57624.3387 & -39.90 & 0.34 & 21 & SL \\
\hline 57625.3144 & -40.33 & 0.36 & 23 & $\mathrm{SL}$ \\
\hline 57626.3263 & -40.63 & 0.35 & 34 & SL \\
\hline 57641.3165 & -40.47 & 0.37 & 34 & SL \\
\hline 57643.3638 & -40.92 & 0.36 & 37 & SL \\
\hline 57657.2555 & -40.98 & 0.35 & 28 & SL \\
\hline 57874.4172 & -38.60 & 0.48 & 17 & Ond \\
\hline 57884.4760 & -40.78 & 0.28 & 34 & Ond \\
\hline 57888.5807 & -40.36 & 0.40 & 18 & SL \\
\hline 57901.5031 & -41.40 & 0.38 & 18 & SL \\
\hline 57906.4804 & -39.11 & 0.36 & 14 & $\mathrm{SL}$ \\
\hline 57926.3788 & -40.10 & 0.21 & 27 & Ond \\
\hline 57935.4804 & -40.80 & 0.23 & 24 & Ond \\
\hline 57950.3847 & -40.33 & 0.30 & 15 & Ond \\
\hline 57952.4047 & -40.20 & 0.21 & 50 & Ond \\
\hline 57967.4011 & -40.63 & 0.41 & 23 & SL \\
\hline 57968.3506 & -38.67 & 0.34 & 20 & $\mathrm{SL}$ \\
\hline 57969.4190 & -40.30 & 0.24 & 16 & Ond \\
\hline 57971.4031 & -39.14 & 0.38 & 18 & $\mathrm{SL}$ \\
\hline 57983.3749 & -38.89 & 0.19 & 38 & Ond \\
\hline 57988.3695 & -39.60 & 0.26 & 12 & Ond \\
\hline 57989.3711 & -40.24 & 0.36 & 32 & SL \\
\hline 57995.3298 & -40.76 & 0.19 & 44 & Ond \\
\hline 58015.2968 & -38.10 & 0.35 & 33 & Ond \\
\hline 58017.2932 & -39.36 & 0.34 & 21 & Ond \\
\hline 58036.2559 & -41.19 & 0.35 & 24 & SL \\
\hline 58202.6574 & -40.47 & 0.37 & 25 & SL \\
\hline 58217.6144 & -40.72 & 0.34 & 31 & $\mathrm{SL}$ \\
\hline 58229.5715 & -40.55 & 0.35 & 39 & SL \\
\hline 58245.5763 & -39.92 & 0.38 & 37 & SL \\
\hline 58249.5893 & -40.01 & 0.41 & 22 & SL \\
\hline 58252.5602 & -40.69 & 0.36 & 26 & SL \\
\hline 58319.4683 & -40.17 & 0.21 & 37 & Ond \\
\hline 58320.4124 & -40.77 & 0.21 & 44 & Ond \\
\hline 58326.4110 & -39.53 & 0.23 & 33 & Ond \\
\hline 58334.4223 & -38.96 & 0.24 & 37 & Ond \\
\hline 58343.3805 & -40.56 & 0.38 & 33 & SL \\
\hline 58347.3279 & -41.67 & 0.18 & 39 & SP \\
\hline 58347.3360 & -40.34 & 0.39 & 26 & SL \\
\hline 58347.4378 & -39.17 & 0.21 & 30 & Ond \\
\hline 58351.3182 & -40.34 & 0.20 & 34 & Ond \\
\hline 58352.3063 & -40.40 & 0.21 & 34 & Ond \\
\hline 58357.3184 & -40.58 & 0.24 & 22 & Ond \\
\hline 58358.3320 & -40.24 & 0.38 & 26 & SL \\
\hline 58361.3259 & -42.14 & 0.20 & 17 & $\mathrm{SP}$ \\
\hline 58361.3517 & -40.87 & 0.37 & 30 & SL \\
\hline 58366.3023 & -41.33 & 0.27 & 33 & Ond \\
\hline 58374.2994 & -40.40 & 0.38 & 32 & SL \\
\hline 58379.3391 & -40.00 & 0.38 & 35 & SL \\
\hline 58380.3073 & -40.95 & 0.38 & 32 & SL \\
\hline 58388.4004 & -39.12 & 0.24 & 47 & Ond \\
\hline 58392.2790 & -40.09 & 0.38 & 29 & SL \\
\hline 58403.2579 & -42.13 & 0.19 & 48 & SP \\
\hline 58427.2124 & -40.39 & 0.18 & 34 & SP \\
\hline
\end{tabular}

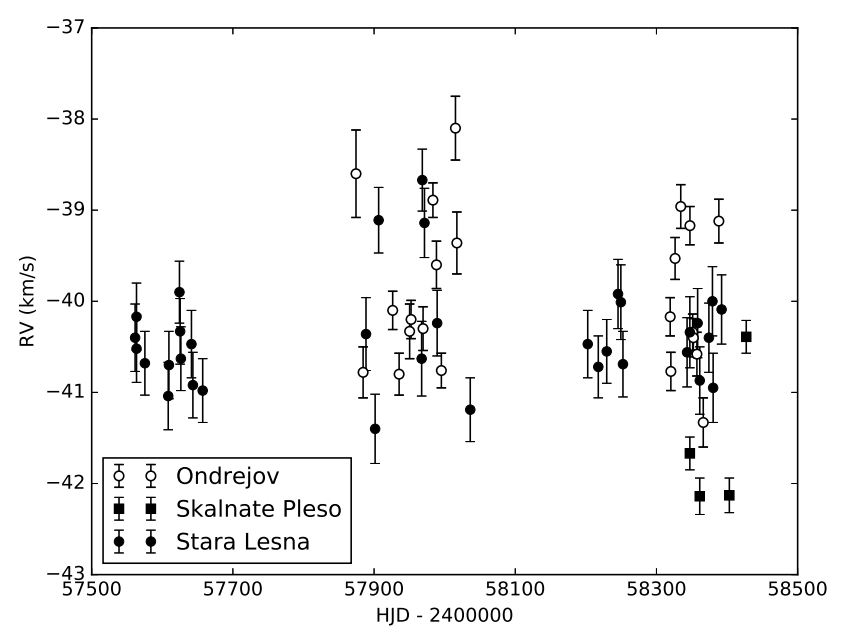

Figure 2. Radial velocities of Kepler-410.

spectrum of $\sigma$ Boo (F4V spectral type star with V magnitude 4.47) observed at Skalnaté Pleso Observatory on 31 May 2018. Radial velocities were corrected according to heliocentric correction and radial velocity of $\sigma$ Boo $(370 \mathrm{~m} / \mathrm{s}$; Nidever et al. 2002). All our measurements are listed in Table 1 and displayed on Figure 2 .

We did not observe any periodic variation on a radialvelocity curve with an amplitude greater than $\sim 600-$ $800 \mathrm{~m} / \mathrm{s}$ over observing period. All obtained RVs are distributed close around their mean value $-40.3 \mathrm{~km} / \mathrm{s}$. A few outlier points are caused by poor quality of these spectra. We also observed the bigger variance of the data obtained from Stará Lesná observatory in 2017 comparing to the data from the same observatory in different years. It could be caused by some systematic error during the observations (e.g. problem with the thermal stability of spectrograph). The relatively big scatter of data collected on Ondrejov observatory could be caused by the slightly different methodology of observations and spectra reduction than in both Slovak observatory. Possible reduction of these (mainly) systematic variations would decrease the maximal amplitude of hypothetical RV signal by about $100 \mathrm{~m} / \mathrm{s}$.

Multi-star explanation of observed TTVs, which would lead to RV with an amplitude at the level $\sim 25-30 \mathrm{~km} / \mathrm{s}$, seems to be very unlikely. We can also exclude the existence of a brown dwarf (down to mass $\approx 30 \mathrm{M}_{J u p}$ ) on the nearly edge-on orbits (i.e. the inclination angle is $90^{\circ}$ ) with period up to 2 years and massive hot Jupiter in this system. The presence of hot Jupiter is of course excluded by the absence of its transits and by the transit timing of Kepler-410Ab (see Figure 3). Unfortunately, the precision of our RV measurements is not enough to put this mass constraint below that of given by TTVs analysis.

\section{MEAN-MOTION RESONANCES}

Another possible explanation of periodic TTVs could be resonant perturbations between transiting planet Kepler$410 \mathrm{Ab}$ and another (unknown) planet with low mass (at the level of Earth mass) in this system. Resonant interactions are quite frequent among exoplanetary systems (e.g. Fabrycky 


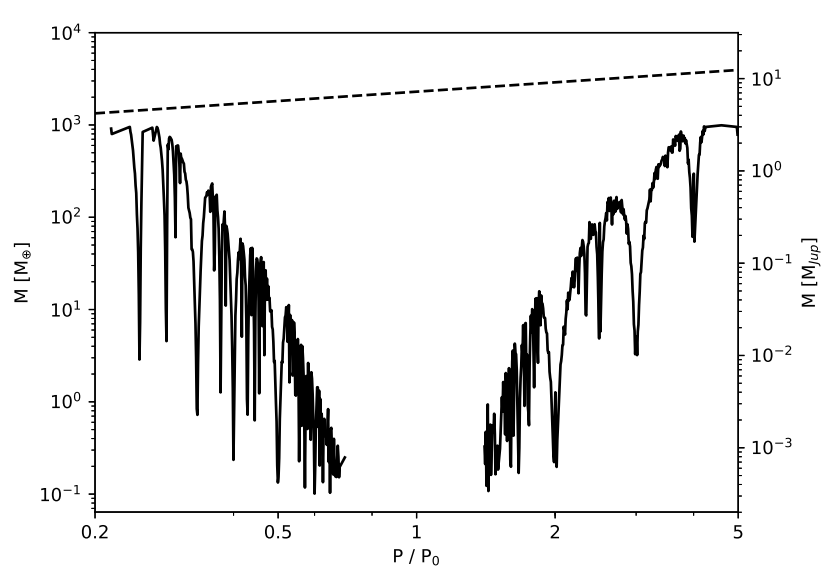

Figure 3. The upper-mass limits of a hypothetical additional planet in the Kepler-410 system based on TTV analysis (solid curve) and RV measurements (dashed line).
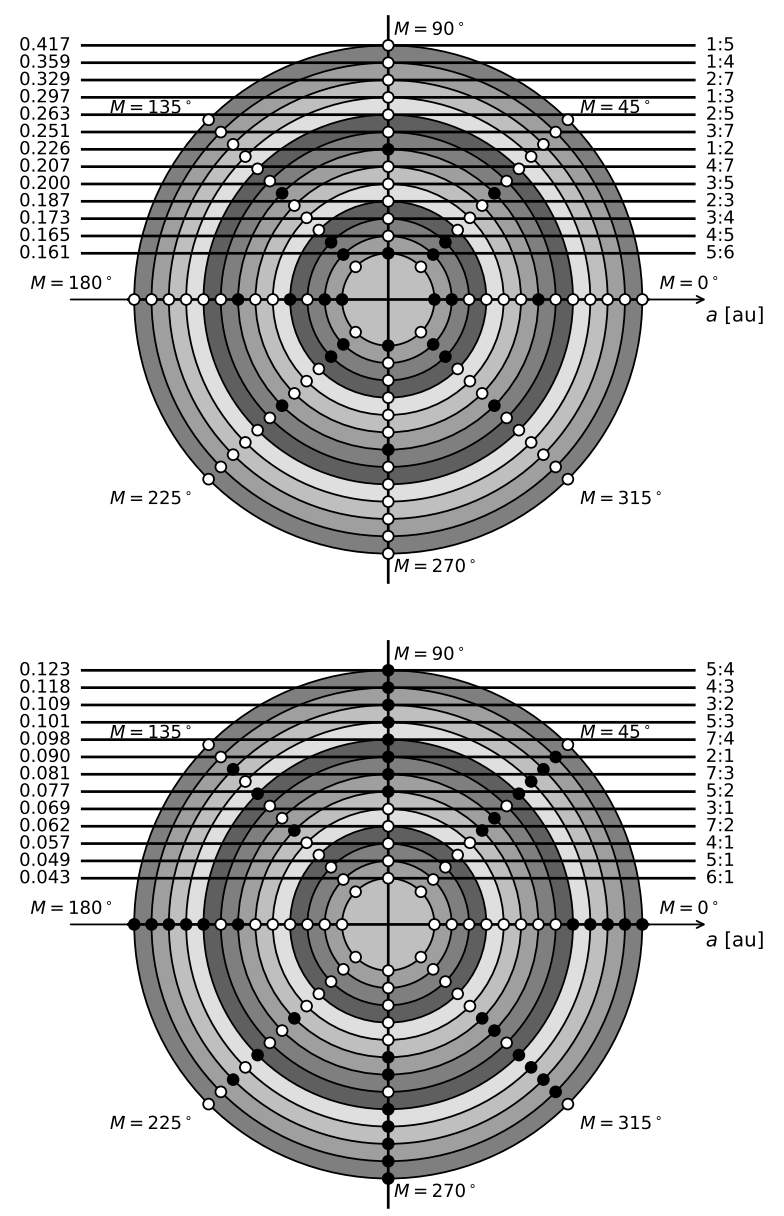

Figure 4. Stability of resonances for different values of initial mean anomaly $M$. Top: exterior resonances. Bottom: interior resonances. White points indicate stable motion; black points show unstable motion. et al. 2012). Wang \& Ji (2014) reported that mean-motion resonances (MMRs) 1:2 and 2:3 are most common in the systems observed by Kepler.

We used a method of upper mass limit Gibson et al. 2009 to determine the mass of a possible perturber. We set the amplitude of the observed TTVs as the amplitude of possible TTVs caused by a perturber. We assumed that the orbit of perturbing planet is circular and coplanar with that of transiting planet. We used Chamber's MERCURY6 code (Chambers 1999) to produce 20000 synthetic O-C diagrams for different configurations of mass and an orbital period of a hypothetical planet. We applied the Bulirsch-Stoer algorithm (Stoer \& Bulirsch 1980) to solve our three-body problem. Our calculation covered the whole length of the observing period ( 1000 days). The results of our simulation are in Figure 3 It displays the upper-mass limit of a hypothetical perturbing planet as a function of the ratio of the orbital period of perturbing planet $(P)$ and that of the transiting planet Kepler-410Ab $\left(P_{0}\right)$. Regions around $P / P_{0} \approx 1$ were found to be unstable due to frequent close encounters of the planets. Value of the upper mass limit is, in our case, the exact mass of possible planet orbiting with the period $P$. The existence of any planet above this limit is excluded by the amplitude of observed TTVs.

We could use the equation by Deck \& Agol (2016):

$$
P_{T T V}=\frac{1}{\left|j / P-(j-n) / P_{0}\right|}
$$

to calculate the the period of the perturbing planet where $P_{T T V}$ is a period of TTVs. We assume exterior MMRs $P_{0}: P \approx j-n: j$ where $j$ and $n$ are small integer numbers and $j>n \geq 1$. Using this equation and the results shown in Figure 3 we could determine the mass and period of perturbing planet which generates TTV signal with a shape similar to the observed one displayed in Figure 1 It is possible to find the solution for all kinds of resonances and we do not have any information about which resonance is correct.

Following Freistetter et al. (2009), we studied the stability of planets inside MMRs with Kepler-410Ab. In this simulation, we placed massless test particles directly inside a resonance. We performed this calculation for different interior $\left(P<P_{0}\right)$ and exterior $\left(P>P_{0}\right)$ resonances and different values of initial mean anomaly $M$. After $10^{5}$ revolutions of Kepler-410Ab, we analysed the stability of all resonances on a long-time scale.

Our results of stability of MMRs are shown in Figure 4 The exterior resonances are in general more stable than the interior. Only interior resonances with a higher resonance ratio are stable. Comparing the statistical representation of each resonance among the other exoplanetary systems (Wang \& Ji 2014) and our stability study, the exterior resonance 2:3 with Kepler-410Ab looks the most probable. The planet with mass $\sim 1.5 \mathrm{M}_{\text {Mars }}$ and orbital period $\sim 26.5$ days (close to the resonance 2:3) generates the TTV signal with a shape similar to the observed one. This planet would also be transiting (for coplanar orbits) but the depth of these transits (at a level of $0.01 \mathrm{mmag}$ ) is difficult to observe with current ground-based telescopes. We found no sign of transits of an additional planet in the Kepler-410 system in data from Kepler mission. The precision of Kepler is probably not sufficient to observe this very shallow transit. We believe that supposed precision of space missions Plato 
and CHEOPS (about 10 ppm; Marcos-Arenal et al. 2014. Fortier et al. 2014) would allow detecting also such small exoplanets.

A hypothetical planet with a higher resonance ratio to Kepler-410Ab would be larger and produce a deeper transit. However the probability of such resonance is smaller and so is the probability of observable transit. A quite common MMR 1:2 seems to be unstable in the system Kepler- 410 .

\section{LONG-TERM STABILITY}

In this section, we study the long-term gravitational influence of the transiting planet on another potential planet in the system. We performed numerical simulations of the system with the parent star Kepler-410A, the transiting planet Kepler-410Ab and a large number of massless particles representing the hypothetical lower mass (earth mass or smaller) planets. The dwarf star Kepler-410B is too far to influence the stability of the planets on studied orbits.

We have used the method of the maximum eccentricity by Dvorak et al. (2003) to generate the stability maps. The value of maximum eccentricity of tested particle gives us information about the probability of a close encounter between the studied body and a massive planet Kepler-410Ab. An orbit with many close encounters will be, of course, unstable in the long term. Other authors (e.g. Freistetter et al. 2009) have noted that the maximum eccentricity is a good indicator of the stability of the orbit.

We performed numerical integration of the orbits for $10^{5}$ revolutions of Kepler- $410 \mathrm{Ab}$, giving a time span of approximately 4500 years. We again used the Bulirsch-Stoer algorithm and MERCURY6 code. The parameter $\epsilon$ which controls the accuracy of the integration was set to $10^{-8}$.

We adopted the parameters of Kepler-410Ab from Van Eylen et al. (2014) for our simulations. We assumed eccentric orbit of Kepler-410Ab with eccentricity 0.17 (Van Eylen et al. 2014). The mass of Kepler-410Ab is unknown. We estimated its mass using the known radius and the massradius relation $R \sim M^{0.55 \pm 0.02}$ (up to $\sim 100 \mathrm{M}_{\oplus} ;$ Bashi et al. 2017). The calculated mass of Kepler-410Ab is $\sim 6 \mathrm{M}_{\oplus}$. To determine an accurate mass of Kepler- $410 \mathrm{Ab}$, one would need RV measurements with precision $1 \mathrm{~m} / \mathrm{s}$.

We have generated $3 \times 10^{5}$ massless particles with semimajor axes ranging from 0.01 au to 0.71 au ( 5 times the semimajor axis of Kepler-410Ab). The inner border was less than 2 times the star radius. The step for the semi-major axis was set to 0.0014 au (one hundredth of the semi-major axis of Kepler-410Ab). The upper limit of eccentricity was 0.5 and the step was 0.02 . The mutual inclination of orbits of a test particle and the orbit of Kepler-410Ab was investigated from $0^{\circ}$ to $50^{\circ}$ with the step of $1^{\circ}$. Other orbital parameters (e.g. mean anomaly) were fixed to 0 in this simulation.

Figure 5 shows the stability maps in the $a-e$ plane for Kepler-410A system for selected values of inclination: $i=0^{\circ}, i=10^{\circ}, i=20^{\circ}, i=30^{\circ}, i=40^{\circ}, i=50^{\circ}$. The initial inclination of the orbit has minimal influence on the shape of the stability map in the $a-e$ plane. It plays some role in the case of orbits closer to the parent star. Inner orbits are also stable up to inclinations of $30^{\circ}$. On the other hand, the impact of the initial eccentricity on the stability of the orbit is important. The shape of unstable regions is similar to a double cone with the apex at the assumed eccentricity $(e=$ 0.17 ) of Kepler-410Ab. The other objects with a different value of eccentricity cross the orbit of Kepler-410Ab also on orbits with the semi-major axis which differ more from that of Kepler-410Ab. And it causes a higher probability of a close encounter with the giant planet. The biggest number of possible orbits is therefore for eccentricities $e \approx 0.17$.

In Figure6, we present stability maps in the $a-i$ plane for the system for selected initial values of eccentricity: $e=$ $0.0, e=0.1, e=0.17, e=0.2, e=0.3, e=0.4, e=0.5$. The width of the stable regions is generally almost independent of the inclination, mainly for lower values of eccentricity. For higher values of eccentricity, there is a wider unstable region for the lower inclinations.

We also studied effect of the initial mean anomaly on stability. We generated the stability maps in the $a-e$ plane for selected values of the mean anomaly: $M=0^{\circ}, M=$ $45^{\circ}, M=90^{\circ}, M=135^{\circ}, M=180^{\circ}$. We were interested only in coplanar orbits (i.e. $i=0^{\circ}$ ). Results of this simulation are shown in Figure 7. The last picture on this figure was created by taking the global maximum eccentricity over all values of the initial mean anomaly. In all cases, the shape of the stable regions looks the same, i.e. it is not influenced by the mean anomaly. However, additional stable and unstable regions corresponding with the MMRs are strongly dependent on the initial mean anomaly.

\section{CONCLUSIONS}

We performed spectroscopic observations of Kepler-410 during three years. In all obtained spectra, we detected spectral lines only from one source (Kepler-410A). We made 60 RV measurements. We did not observe any periodic variation of the radial velocities with amplitude more than $800 \mathrm{~m} / \mathrm{s}$ over three observing seasons. Therefore, we rejected our previous hypothesis that observed TTVs are caused by the presence of another stellar companion in the system Gajdoš et al. 2017). We can also exclude the existence of a brown dwarf (or any other more massive object) down to a mass of about $30 M_{\text {Jup }}$ on the orbits with period up to 2 years or a massive hot Jupiter in the Kepler-410 system based on the accuracy of our RV measurements on nearly edge-on orbits.

Another logical explanation of the observed TTVs is gravitational interaction with an additional planet orbiting near to the MMR. Our analysis of the stability of the most common interior and exterior resonances shows that the exterior MMR 2:3 is the most probable. Small planet $\left(\sim 1.5 \mathrm{M}_{\text {Mars }}\right)$ on an orbit with period $\sim 26.5$ days (close to resonance 2:3) would cause a similar TTVs as observed in Kepler-410Ab. Unfortunately, this planet would be so small that its observational evidence is hardly possible to get at present time. This additional planet would be transiting with depth of transit at the level of only $0.01 \mathrm{mmag}$. The radial-velocity measurements with precision $1 \mathrm{~cm} / \mathrm{s}$ would be required to detect this planet spectroscopically. The successful observation would be also complicated by the stellar oscillations of Kepler-410A (Van Eylen et al. 2014). However, prepared space missions Plato and CHEOPS should detect also similarly small exoplanets.

Finally, we investigated the dynamical stability of the system in order to identify stable regions where additional 

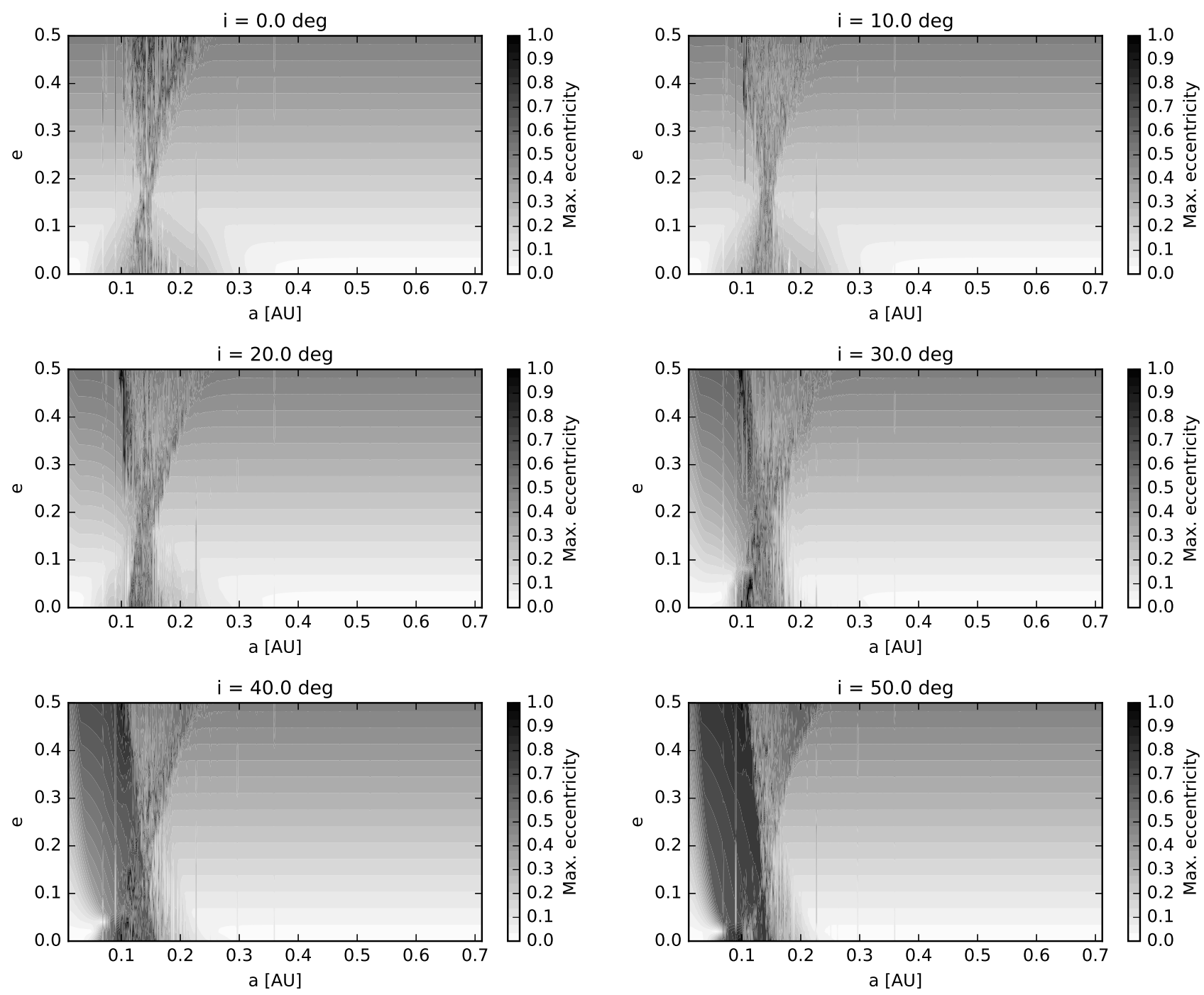

Figure 5. Stability plot in the $a-e$ plane for different values of $i$ showing the maximum eccentricity (as an indicator of the stability of the orbit) for the system Kepler-410. From top left to bottom right: $i=0^{\circ}, i=10^{\circ}, i=20^{\circ}, i=30^{\circ}, i=40^{\circ}, i=50^{\circ}$.

planets could exist for a long time (hundreds of years). We found that a hypothetical planet could exist relatively close to the known transiting planet, mainly on coplanar orbit with eccentricity similar to that of known planet Kepler$410 \mathrm{Ab}(e=0.17)$. Raising or reduction of the eccentricity from this value causes the unstable region to start to grow. Orbits with semi-major axis more than $\sim 0.25$ au are stable for almost all combinations of eccentricities and inclinations (except some MMRs). The inner orbits are stable for orbital inclinations up to $20^{\circ}-30^{\circ}$.

From a dynamic point of view, the possibility of other planets in the system is high. There exists a wide range of orbits on which such planets could exist for a long time.

\section{ACKNOWLEDGEMENT}

We are grateful to Z. Garai, E. Kundra, E. Paunzen, R. Komžík and P. Sivanič for help in obtaining the observations of Kepler-410 at Observatory Stará Lesná and Skalnaté Pleso. This paper has been supported by the grant of the Slovak Research and Development Agency with number APVV-15-0458 and was created under project ITMS No.26220120029, based on the supporting operational Research and development program financed from the European Regional Development Fund. The research of P.G. was supported by internal grant VVGS-PF-2017-724 of the Faculty of Science, P. J. Šafárik University in Košice. M.V., T.P. and L.H. would like to thank the project VEGA 2/0031/18. P.K., J.S. and D.D. would like to acknowledge the support from GACR international grant 17-01752J. M.S. acknowledges financial support of Postdoc@MUNI project CZ.02.2.69/0.0/0.0/16_027/0008360.

\section{References}

Adams E. R., Ciardi D. R., Dupree A. K., Gautier T. N. I., Kulesa C., McCarthy D., 2012, AJ 144, 42

Agol E., Steffen J., Sari R., Clarkson W., 2005, MNRAS 359, 567 Bashi D., Helled R., Zucker S., Mordasini C., 2017, A\&A 604, A83 

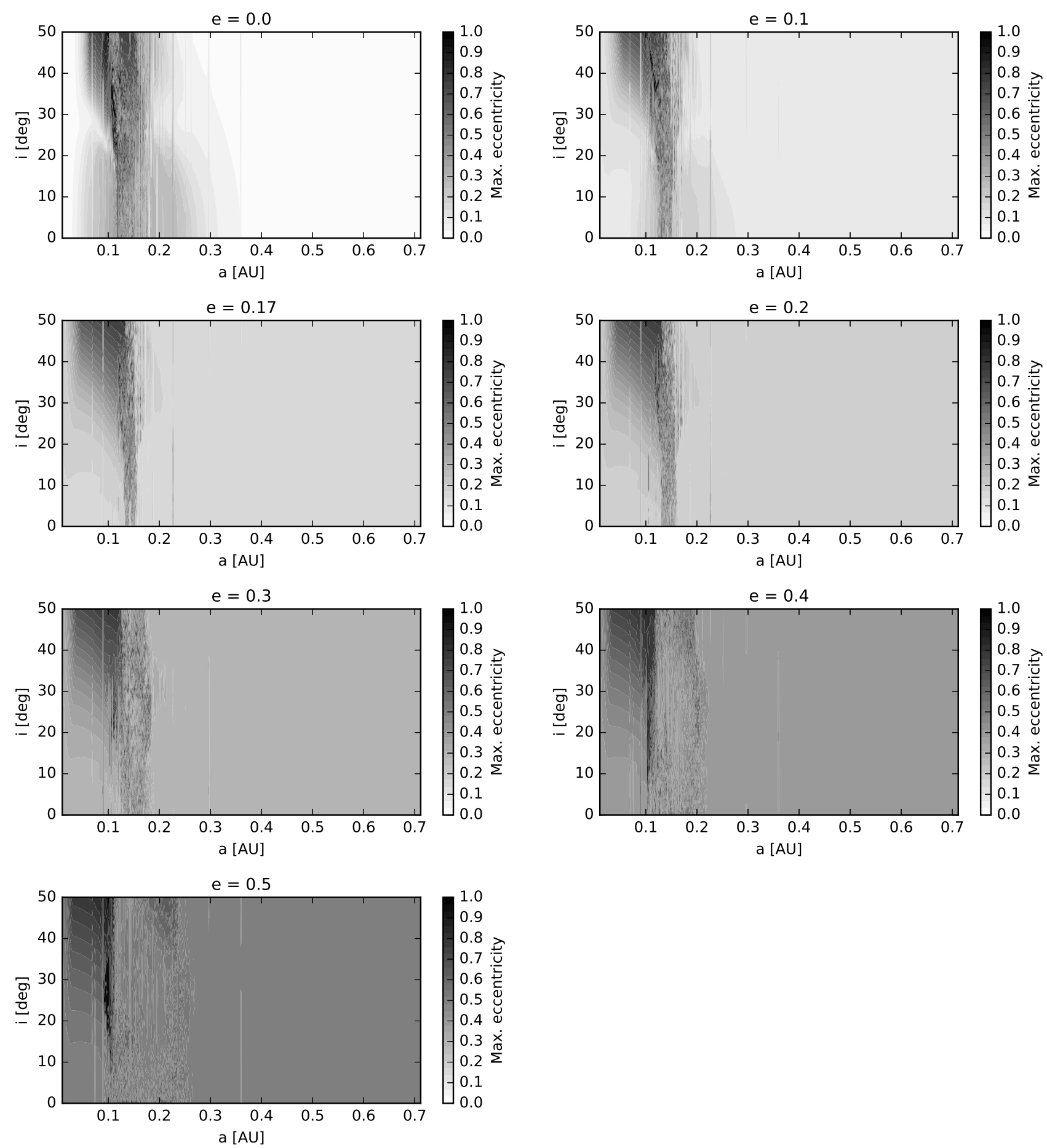

Figure 6. Stability plot in the $a-i$ plane for different values of $e$ showing the maximum eccentricity for the system. From top left to bottom left: $e=0.0, e=0.1, e=0.17, e=0.2, e=0.3, e=0.4, e=0.5$.

Baudrand J., Bohm T., 1992, A\&A, 259, 711

Blanco-Cuaresma S., Soubiran C., Heiter U., Jofré P., 2014, A\&A, 569, A111

Chambers J. E., 1999, MNRAS 304, 793

Deck K. M., Agol E., 2016, ApJ 821, 96

Dvorak R., Pilat-Lohinger E., Funk B., Freistetter F., 2003, A\&A, 398, L1

Fabrycky D. C., et al., 2012, ApJ, 750, 114

Fortier A., Beck T., Benz W., Broeg C., Cessa V., Ehrenreich
D., Thomas N., 2014, in Space Telescopes and Instrumentation 2014: Optical, Infrared, and Millimeter Wave. p. 91432J, doi:10.1117/12.2056687

Freistetter F., Süli Á., Funk B., 2009, Astron. Nachr. 330, 469

Gaia Collaboration et al., 2018, A\&A, 616, A1

Gajdoš P., Parimucha Š., Hambálek L', Vaňko M., 2017, MNRAS. 469, 2907

Gibson N. P., et al., 2009, ApJ 700, 1078

Holczer T., et al., 2016, ApJS 225, 9 

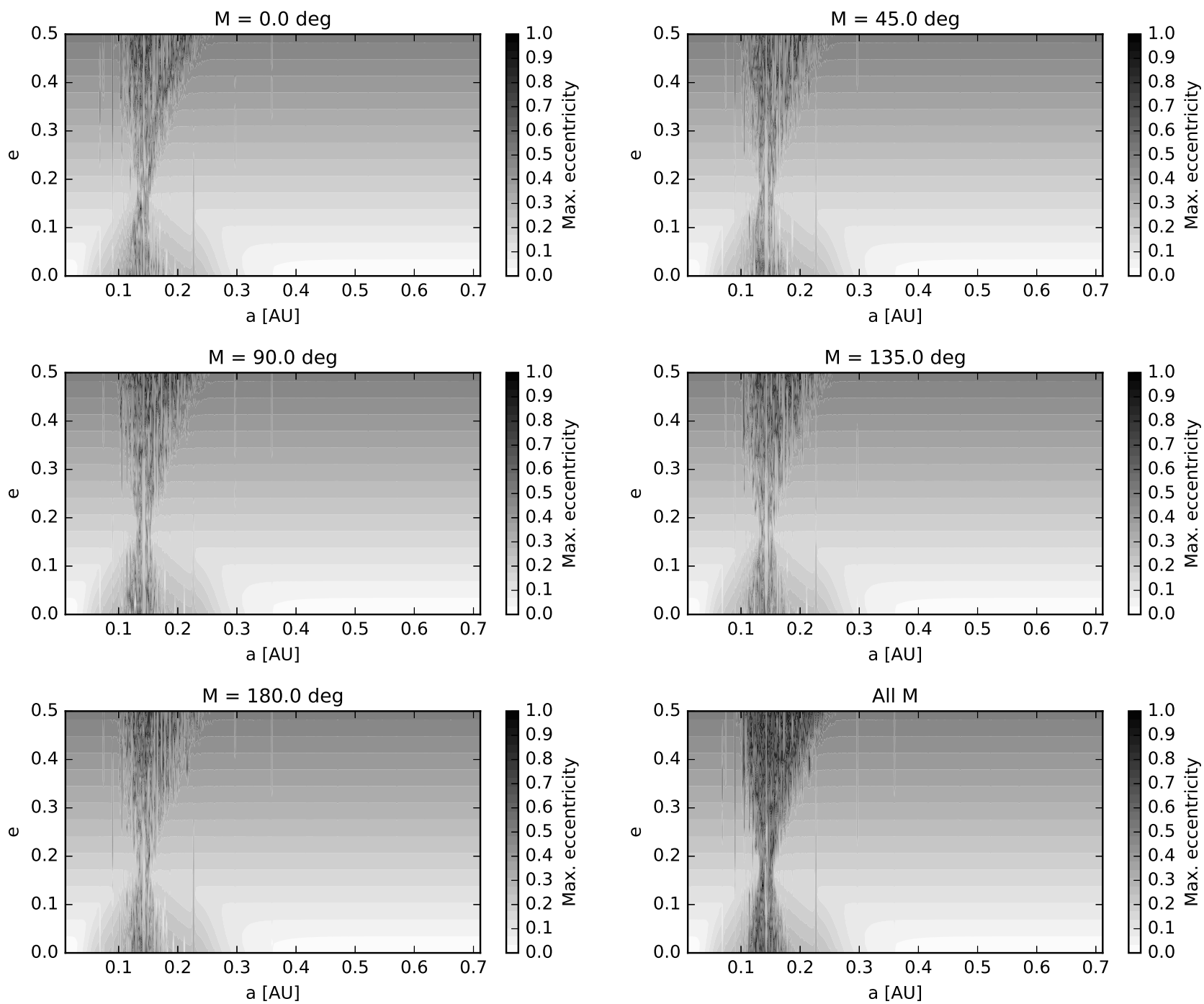

Figure 7. Stability plot in the $a-e$ plane for different values of $M$ showing the maximum eccentricity for the system. From top left to bottom left: $M=0^{\circ}, M=45^{\circ}, M=90^{\circ}, M=135^{\circ}, M=180^{\circ}$. Bottom right: combination of all figures using the maximal value of maximum eccentricity over all values of $M$.

Irwin J. B., 1952a, ApJ 116,211

Irwin J. B., 1952b, ApJ, 116, 218

Kabath P., Skarka M., Sabotta S., 2018, submitted to AJ

Marcos-Arenal P., et al., 2014, A\&A 566, A92

Mazeh T., et al., 2013, ApJS, 208, 16

Molenda-Żakowicz J., et al., 2013, MNRAS 434, 1422

Nidever D. L., Marcy G. W., Butler R. P., Fischer D. A., Vogt S. S., 2002, ApJS 141, 503

Pribulla T., et al., 2015, Astron. Nachr., 336, 682

Stoer J., Bulirsch R., 1980, Introduction to numerical analysis. Springer-Verlag, New York

Tody D., 1993, in Hanisch R. J., Brissenden R. J. V., Barnes J., eds, Astronomical Society of the Pacific Conference Series Vol. 52, Astronomical Data Analysis Software and Systems II. p. 173

Van Eylen V., et al., 2014, ApJ 782, 14

Wang S., Ji J., 2014, ApJ 795, 85 